\title{
Caracterización de dientes en una población con tratamiento de endodoncia atendida en una clínica odontólogica universitaria mediante tomografía cone beam y comparación con radiografía periapical
}

Characterization of teeth in a population with endodontic treatment attended in a university dental clinic using cone beam tomography and comparison with periapical radiography

\section{Caracterização dos dentes de uma população em tratamento endodôntico atendida em clínica odontológica universitária por meio de tomografia cone beam e comparação com radiografia periapical}

\author{
Eliana Pineda Vélez ${ }^{1}$ \\ Samantha Bedoya Morales ${ }^{2}$ \\ Frank David Echeverri Romero ${ }^{2}$ \\ Yeison Andrés Guerra Bedoya ${ }^{3}$ \\ Luisa Osorno Jaramillo 4 \\ John Querubín Franco Aguirre ${ }^{5}$
}

Recibido: 30 de septiembre de 2020

Aprobado: 30 de octubre de 2020

Publicado: 15 de diciembre de 2020

Cómo citar este artículo:

Pineda-Vélez E, Bedoya-Morales S, Echeverri-Romero FD, Guerra-Bedoya YA, Osorno-Jaramillo L, Franco-Aguirre JQ. Caracterización de dientes en una población con tratamiento de endodoncia atendida en una clinica odontologica-universitaria mediante tomografia cone-beam y comparación con radiografía periapical. Revista Nacional de Odontología. (2020); 16(2), 1-13. doi: https://doi.org/10.16925/2357-4607.2020.02.02

Artículo de investigación. https://doi.org/10.16925/2357-4607.2020.02.02

$1 \quad$ MsC en Epidemiología, Especialista en Endodoncia. Universidad Cooperativa de Colombia. Universidad de Antioquia, Medellín, Colombia. Facultad de Odontología de la Universidad Cooperativa de ColombiaCarrera $13 \mathrm{~N}^{\circ}$. 37-43 octavo piso. Bogotá, Colombia.

Correo electrónico: elipineda1@gmail.com.

ORCID: https://orcid.org/0000-0002-2431-7489

2 Odontologos, Universidad Cooperativa de Colombia.

ORCID: https://orcid.org/0000-0002-3057-4940

ORCID: https://orcid.org/0000-0001-6600-1477

3 OD IPS Centro Odontológico San Pedro, Medellín, Colombia.

ORCID: https://orcid.org/0000-0002-8878-8060

$4 \quad$ OD, Radióloga Oral y Maxilofacial. Universidad Cooperativa de Colombia, Medellín, Colombia. ORCID: https://orcid.org/0000-0002-9309-9163

5 Microbiólogo y Bioanalista: Msc Microbiología. Profesor Universidad Cooperativa de Colombia, Facultad de Odontología y Universidad de Antioquia, Escuela de Microbiología, Medellín, Colombia. ORCID: https://orcid.org/0000-0003-1592-2916 
2 Caracterización de dientes en una población con tratamiento de endodoncia atendida en una clínica odontológica universitaria mediante tomografía cone beam y comparación con radiografía periapical

\section{Resumen}

Introducción: las imágenes que proporcionan las radiografias son en dos dimensiones. Esto no permite en muchas ocasiones una visualización real de las condiciones anatomicas de los dientes y especialmente de los tejidos periapicales. Es por esto que la tomografia cone beam puede proporcionar una imagen que permita identificar mejor el estado de los dintes y su respuesta a la terpaia endodontica.

Objetivo: caracterizar dientes en una población con tratamiento de mediante tomografia cone beam y comparación con radiografía periapical.

Materiales y medotods: estudio descriptivo transversal, en el cual se realizó un examen clínico detallado, se tomó la radiografía y tomografía cone beam de los pacientes que previamente habían sido tratados con endodoncia, Se realizó un análisis descriptivo de la información. Se evalúo la concordancia, 0,56.

Resultados: se evidencia que la frecuencia de lesión por tomografia fue de $29 \%$. Mientras que el diagnostico de lesión radiográfica se presentó en el 19,4\% de los dientes analizados. Se encuentra además que la mayor frecuencia de lesión reportada por tomografia se presentó en dientes con obturaciones incompletas, de la misma forma que en mayor en dientes multirradiculares,

Conclusion: la tomografia cone beam es una herramienta que aun no es muy utilizada ni reconocida por los clínicos en su potencial diagnostico, y de plan de tratamientos de casos endodonticos.

Palabras clave: endodoncia, radiografia, cone beam, anatomia radicular, diagnóstico.

\section{Abstract}

Introduction: The images provided by radiographs are in two dimensions, which in many cases does not allow a real visualization of the anatomical conditions of the teeth and especially of the periapical tissues. This is why cone beam tomography can provide an image that allows better identification of the state of the teeth and their response to endodontic terpaia.

Objective: To characterize teeth in a population treated by cone-beam tomography and comparison with periapical radiography.

Materials and methods: A descriptive cross-sectional study, in which a detailed clinical examination was carried out, radiography and cone beam tomography were taken of patients who had previously been treated with endodontics, a descriptive analysis of the information was carried out, and concordance was evaluated, 0.56.

Results: It is evidenced that the frequency of lesion by tomography was $29 \%$ while the diagnosis of radiographic lesion was presented in $19.4 \%$ of the analyzed teeth, it is also found that the highest frequency of injury reported By tomography it was presented in teeth with incomplete fillings, in the same way as in older ones in multiradicular teeth.

Conclusion: cone beam tomography is a tool that is not yet widely used or recognized by clinicians in its diagnostic potential, and as a plan of endodontic case treatments

Key words: endodontics, radiography, cone beam, root anatomy, diagnosis.

\section{Resumo}

Introdução: as imagens fornecidas pelas radiografias são em duas dimensões. Muitas vezes, isso não permite uma visualização real das condições anatômicas dos dentes e principalmente dos tecidos periapicais. É por isso que a tomografia de feixe cônico pode fornecer uma imagem que permite melhor identificação do estado dos dintes e sua resposta à terpaia endodôntica. 
Eliana Pineda Vélez, Samantha Bedoya Morales, Frank David Echeverri Romero, 3 Yeison Andrés Guerra Bedoya, Luisa Osorno Jaramillo, John Querubín Franco Aguirre Objetivo: caracterizar os dentes em uma população tratada com tomografia de feixe cônico e comparação com a radiografia periapical.

Materiais e métodos: estudo descritivo transversal, no qual foi realizado exame clínico detalhado, radiografia e tomografia de feixe cônico de pacientes previamente tratados com endodontia, foi realizada análise descritiva das informações. A concordância foi avaliada, 0,56.

Resultados: evidencia-se que a frequência de lesão pela tomografia foi de $29 \%$. Já o diagnóstico de lesão radiográfica foi apresentado em $19,4 \%$ dos dentes analisados. Verifica-se também que a maior frequência de lesão relatada pela tomografia ocorreu em dentes com obturações incompletas, da mesma forma que em maior frequência em dentes multirradiculares.

Conclusão: a tomografia de feixe cônico é uma ferramenta ainda pouco utilizada ou reconhecida pelos clínicos por seu potencial para diagnóstico e plano de tratamento de casos endodônticos.

Palavras-chave: endodontia, radiografia, feixe cônico, anatomia radicular, diagnóstico.

\section{Introducción}

El uso de ayudas diagnosticas es trascendental para el manejo de los problemas endodónticos. Las imágenes proporcionadas por las radiografías convencionales ofrecen información limitada dado que sólo son la representación bidimensional de estucturas con ubicación en los tres planos del espacio. Además, aunado a la superposición de estructuras anatómicas adyacentes, supone inconvenientes para su validez diagnostica. Teniendo en cuenta la anatomía de los conductos radiculares y la presencia de fisuras y calcificaciones en estas estructuras, su descripción e identificación supone el empleo de métodos con un grado de exactitud confiable, dentro los cuales la observación de las imágenes en las tres dimensiones a través de la tomografía cone beam es una alternativa de gran validez (1).

La evolución de la imageneologia convencional de dos dimensiones a las imágenes tridimensionales, ha implicado multiples beneficios para el paciente y el profesional de la endodoncia, entre los cuales está la posibilidad de un diagnostico más acertado y en consecuencia un plan de tratamiento con mayores probabilidades de éxito.

La tomografia cone beam es una técnica imagenologica que visualiza las estructuras internas del paciente mediante la emisión de una fuente de rayos $\mathrm{X}$ de penetración en forma de cono, recepcionada por la matriz de las células detectoras. Genera una líneas de respuesta en el proceso conocido como adquisición, posteriormente se genera una reconstrucción de las imágenes a través del procesamiento de la información mediante un software, integrado al equipo emisor de rayos $\mathrm{X}$. La forma conica del rayo proporciona multiples ventajas como el control del tamaño del haz, el cual se ajusta a la zona de interés mediante un colimador que evita la radiación dispersa. Ademas, la resolución espacial es mas uniforme comparada con otros sistemas, lo 
4 Caracterización de dientes en una población con tratamiento de endodoncia atendida en una clínica odontológica universitaria mediante tomografía cone beam y comparación con radiografía periapical

que proporciona mayor nitidez en el detalle de las imágenes, esto es fundamental en la evaluación de imágenes endodónticas (1).

En relación con lo expuesto, se ha permitido evidenciar la utlidad diagnostica de la tomografía cone beam en condiciones como: reabsorción radicular en las diferentes superficies y tercios radiculares, periodontitis apical en estadios iniciales, fracturas radiculares verticales y horizontales, perforaciones radiculares e identificación de instrumentos separados (2). Como ejemplo de ello, Burgos y colaboradores reportaron un caso de fistula en zona de diente 24 a través de tomografía cone beam, que incicialmente fue reportada como lesión para radicular distal sin causa identificada a través de radiografia periapical (3). Paula-Silva y colaboradores evaluaron la validez de la tomografía cone beam en el diagnostico de periodonditis apical reportando una sensibilidad del 91 \% frente al $77 \%$ con radiografia periapical (4). Miyagaki y colaboradores en un reporte de tres casos de fractura radicular vertical destacan la utilidad de la tomografía en la identificación de las líneas de fractura, inicialmente no identificadas con radiografias periapicales (5).

De lo anterior se evidencia que en la evaluación de casos endodonticos es fundamental contar con una herramienta que proporcione los tres planos del espacio y elimine la sobreproyección de estructuras, como es el caso de la tomografía, considerada como una técnica de gran potencial al permitir la observación en todas sus dimensiones del sistema de conductos radiculares, los tejidos y estructuras anatómicas. A razón de lo anterior, el presente estudio tiene por objetivo principal caracterizar los dientes con tratamentos endodonticos en una muestra de individuos atendidos en una clínica universitaria de odontología. La implementación del uso de la tomografía en el área de la endodoncia configura un horizonte amplio de aplicación, al permitir explorar con gran facilidad los planos axial, sagital y coronal de los elementos en estudio y obtener información valiosa para el diagnóstico, plan de tratamiento y evaluaciones post operatorias (6).

\section{Materiales y métodos}

Se realizó un estudio descriptivo transversal en 27 pacientes que asistieron a la clínica de endodoncia de la facultad de Odontología de la Universidad Cooperativa de Colombia sede Envigado, entre los años 2015 y 2017.

Se excluyeron mujeres en estado de gestación, personas que manifestaran la presencia de alguna enfermedad sistémica no controlada, menores de edad e individuos con dentición decidua. 
Eliana Pineda Vélez, Samantha Bedoya Morales, Frank David Echeverri Romero, 5 Yeison Andrés Guerra Bedoya, Luisa Osorno Jaramillo, John Querubín Franco Aguirre

Recoleccion de la información: a partir de los registros de las historias clínicas se contactaron los pacientes para la explicación de los detalles de la investigación: posibles beneficios, confidencialidad de los resultados y los riesgos por ser un estudio con riesgo mayor que el mínimo. Se entregó un consentimiento informado acorde con lo contempado en el articulo 15 de la resolución número 8430 de 1993 del Ministerio de Salud. El participante en presencia de un testigo lo firmo con cédula y huella, posterior a esto se realizó un examen clínico detallado, se tomó la radiografía y tomografía cone beam y nuevamente el paciente firmó una orden para la autorización de los estudios detallados, posteriormente con los resultados obtenidos de los análisis radiologicos se les informó el estado del diente y las posibles opciones de tratamiento si así lo requirió.

Para la descripción de los hallazgos radiológicos se realizó una evaluación intra e interobservador con un experto a través de la estimación del índice Kappa de Cohen con resultado mayor a 0,75 en todas las observaciones descritas.

\section{Análisis estadístico}

Se realizó un análisis descriptivo de la información a través de la estimación de frecuencias relativas y absolutas para las variables de naturaleza cualitativa y el cálculo de medidas de resumen para las variables de naturaleza cuantitativa.

Se exploró la asociación entre el diagnóstico de lesión según la técnica y el nivel de la obturación identificado. También entre la adaptación de la restauración radiográfica y la clasificación radicular con el diagnóstico de lesión tomógrafica a través de la prueba $\mathrm{Chi}^{2}$ de Pearson. Se evalúo la concordancia entre los métodos diagnósticos a través del índice Kappa de Cohen.

En todos los análisis se tomó un valor P de significación estadística menor a 0,05 realizados a través del software IBM SPSS 25

\section{Resultados}

De las características sociodemográficas observadas en la población de estudio, se estimó un promedio de edad de 45,5 \pm 20,7 años y mayor frecuencia de pacientes del sexo masculino (57,1 \%), nivel de educativo secundaria (64,3 \%) y empleados $(35,7 \%)$. Se obtuvieron radiografías periapicales y tomografia axial computarizada de todos los dientes con tratamiento endodontico, para un total de 31 dientes analizados, en su mayoría dientes posteriores. De los dientes analizados, se presentó una mayor frecuencia del 36, 46 y 47, en relación al tipo de raíz se observó una mayor proporción 
6 Caracterización de dientes en una población con tratamiento de endodoncia atendida en una clínica odontológica universitaria mediante tomografía cone beam y comparación con radiografía periapical

de dientes unirradiculares (38,7\%), respecto al nivel de obturacion, $48 \%$ de las endodoncias se encontraban a nivel apical, 35,5\% subobturadas y 16,1 \% sobreobturadas (tabla 1)

Tabla 1. Caracterización de la los dientes

\begin{tabular}{|c|c|c|c|}
\hline & & Recuento & $\%$ \\
\hline \multirow{13}{*}{ Diente } & 11 & 3 & 9,7 \\
\hline & 12 & 1 & 3,2 \\
\hline & 13 & 1 & 3,2 \\
\hline & 14 & 2 & 6,5 \\
\hline & 15 & 1 & 3,2 \\
\hline & 21 & 3 & 9,7 \\
\hline & 22 & 1 & 3,2 \\
\hline & 25 & 1 & 3,2 \\
\hline & 27 & 3 & 9,7 \\
\hline & 36 & 4 & 12,9 \\
\hline & 41 & 1 & 3,2 \\
\hline & 46 & 4 & 12,9 \\
\hline & 47 & 6 & 19,4 \\
\hline \multirow{5}{*}{ Raiz } & Uniradicular & 12 & 38,7 \\
\hline & Mesial & 8 & 25,8 \\
\hline & Distal & 8 & 25,8 \\
\hline & Palatina & 2 & 6,5 \\
\hline & Vestibular & 1 & 3,2 \\
\hline \multirow{3}{*}{ Nivel de obturación } & A Nivel Apical & 15 & 48,4 \\
\hline & Subobturado & 11 & 35,5 \\
\hline & sobreodturado & 5 & 16,1 \\
\hline
\end{tabular}

Fuente: elaboración propia

La frecuencia de lesión por tomografia fue de 29 \% (figura 1), mientras que el diagnostico de lesión radiográfica se presentó en el 19,4 \% de los dientes analizados (figura 2). La concordancia en el diagnóstico de lesión estimada a través del índice de Kappa de Kohen entre los dos métodos diagnósticos fue de 0,56. Adicionalmente, la tomografía permitió la descripción de otros hallazgos no identificados inicialmente en la radiografia periapical (tabla 2 ). 
Eliana Pineda Vélez, Samantha Bedoya Morales, Frank David Echeverri Romero, 7 Yeison Andrés Guerra Bedoya, Luisa Osorno Jaramillo, John Querubín Franco Aguirre

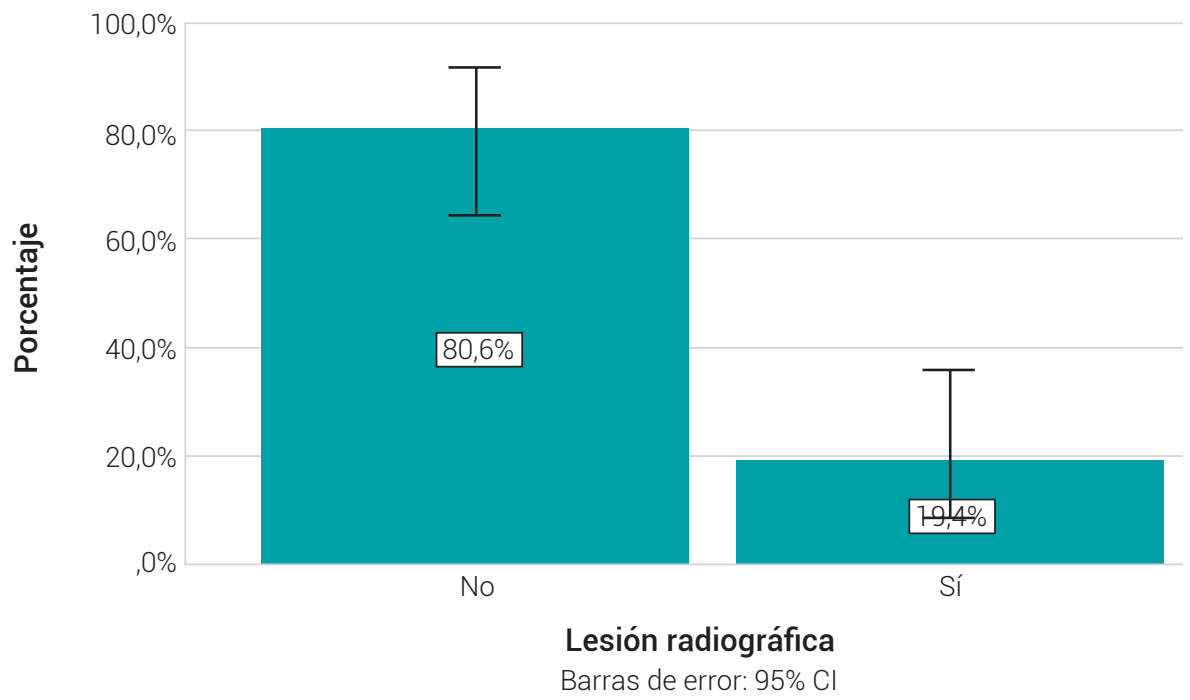

Figura 1. Frecuencia de lesión por radiografia periapical

Fuente: elaboración propia

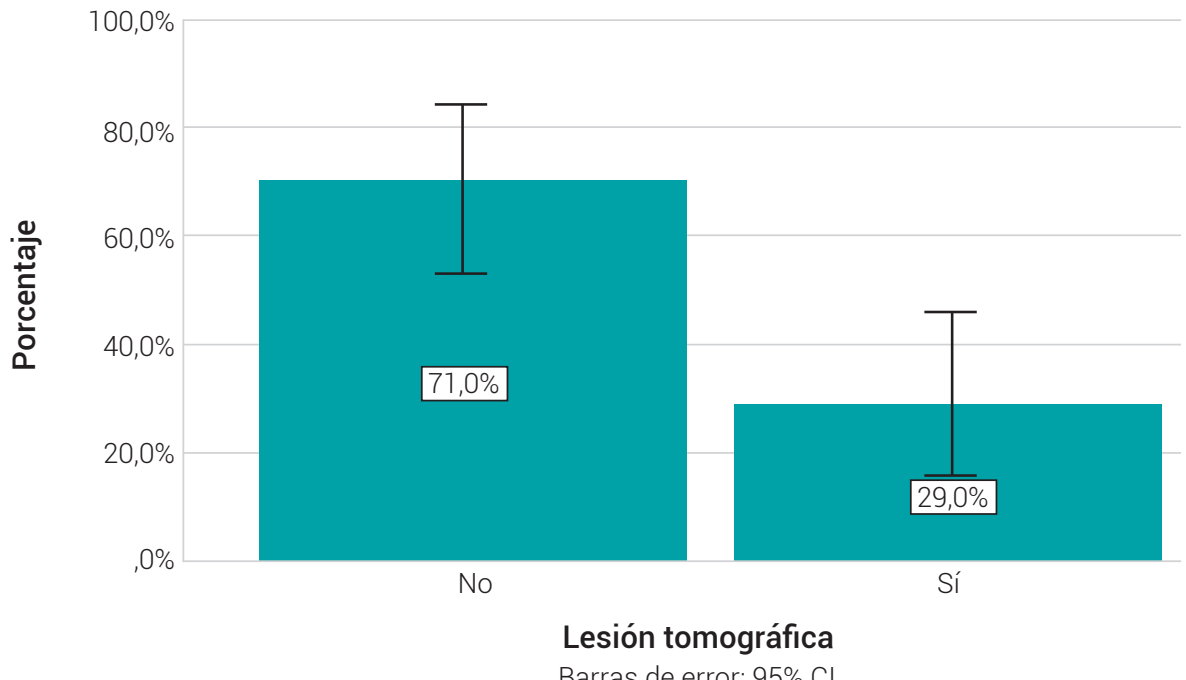

Figura 2. Frecuencia de lesión por tomogracia cone beam

Fuente: elaboración propia 
8 Caracterización de dientes en una población con tratamiento de endodoncia atendida en una clínica odontológica universitaria mediante tomografía cone beam y comparación con radiografía periapical

Tabla 2. Hallazgos radiológicos por tomografía cone beam

\begin{tabular}{|c|c|c|c|}
\hline & & Recuento & $\%$ \\
\hline \multirow{7}{*}{ Hallazgo } & $\begin{array}{l}\text { Marcada reabsorción radicular y erosion de tabla osea } \\
\text { vestibular }\end{array}$ & 2 & 20,0 \\
\hline & Ausencia de corona (Pin intraradicular) & 1 & 10,0 \\
\hline & Conducto con material en rebalse apical & 1 & 10,0 \\
\hline & Erosión a nivel de tercio apical & 1 & 10,0 \\
\hline & $\begin{array}{l}\text { Leve compresión de canal mandibular asociado a zona } \\
\text { de diente } 47\end{array}$ & 3 & 30,0 \\
\hline & $\begin{array}{l}\text { Obturación parcial en longitud en falsa vía a nivel de } \\
\text { tercio apical mesial }\end{array}$ & 1 & 10,0 \\
\hline & $\begin{array}{l}\text { Obturación parcial en longitud en falsa vía que compro- } \\
\text { mete el contorno radicular externo apical mesial }\end{array}$ & 1 & 10,0 \\
\hline
\end{tabular}

Fuente: elaboración propia

Entre los resultados obtenidos se encontró que la frecuencia de lesión tomógrafica estaba asociada con el nivel de obturación del diente $(\mathrm{Vp}$ Chi = 0,025) (tabla 3). En tal sentido, se evidenció que la mayor frecuencia de lesión reportada por tomografia se presentó en dientes subobturados, en tanto que las lesiones reportadas por radiografia presentaron las mismas proporciones en los tres niveles de obturación reportados. La frecuencia de lesión tomografica fue mayor en dientes multirradiculares, pero sin diferencias significativas respecto a la frecuencia de lesión en los unirradiculares (Vp Chi²=0,228) (figura 3).

Tabla 3. Frecuencia de lesión según nivel de obturación del diente

\begin{tabular}{|c|c|c|c|c|c|c|}
\hline & & \multicolumn{2}{|c|}{ Lesión radiográfica } & \multicolumn{2}{|c|}{ Lesión tomográfica } & \multirow{3}{*}{ Vp } \\
\hline & & No & Si & No & Si & \\
\hline & & $\%$ & $\%$ & $\%$ & $\%$ & \\
\hline \multirow{3}{*}{$\begin{array}{l}\text { Nivel de } \\
\text { obturación }\end{array}$} & A Nivel Apical & 52,0 & 33,3 & 63,6 & 11,1 & \multirow{3}{*}{0,025} \\
\hline & Subobturado & 36,0 & 33,3 & 27,3 & 55,6 & \\
\hline & Sobreodturado & 12,0 & 33,3 & 9,1 & 33,3 & \\
\hline
\end{tabular}

Fuente: elaboración propia 
Eliana Pineda Vélez, Samantha Bedoya Morales, Frank David Echeverri Romero, 9 Yeison Andrés Guerra Bedoya, Luisa Osorno Jaramillo, John Querubín Franco Aguirre

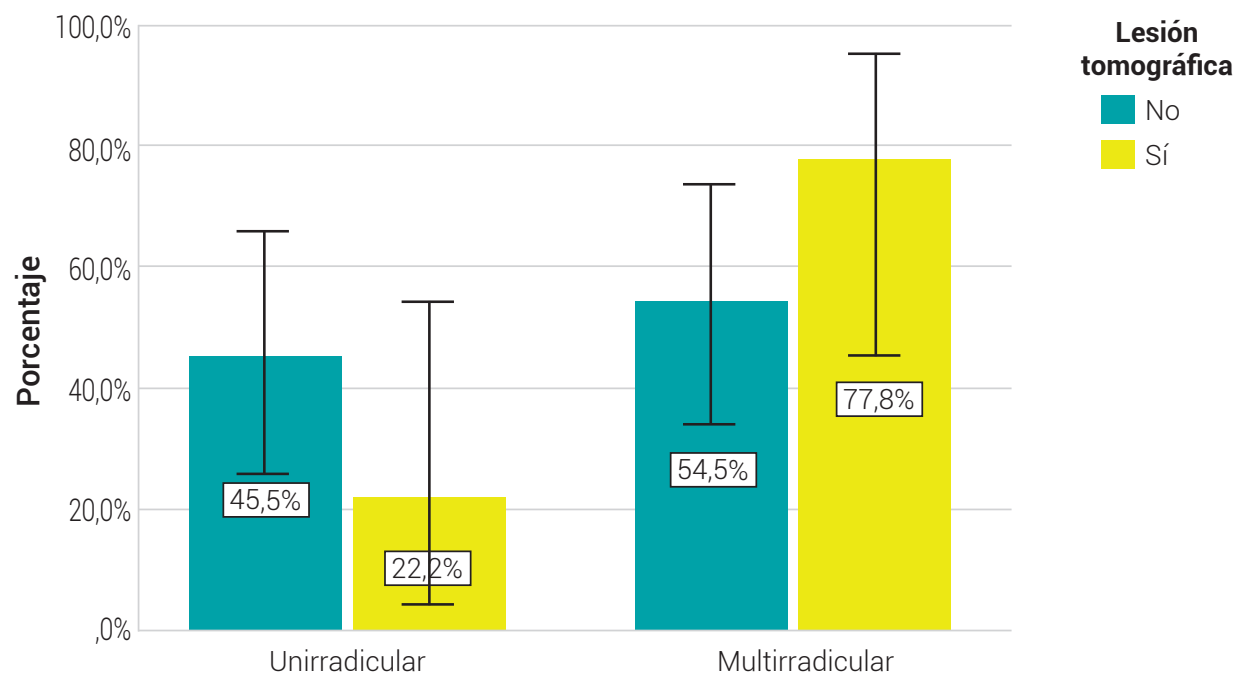

Clasificación de la raíz

Barras de error: $95 \% \mathrm{Cl}$

Figura 3. Frecuencia de lesión tomografica según clasificación de la raíz

Fuente: elaboración propia

\section{Discusión}

Los resultados del presente estudio dejan ver a la tomografia cone beam como una herramienta fundamental en la evaluación de lesiones apicales, por efecto de la representación tridimensional que proporciona de las estructuras del diente y la exclusión de elementos anatómicos que pueden interferir la valoración dental y periapical. Se manifiesta mayor sensibilidad y precisión (6).

La diferencia en la proporción estimada de lesiones apicales, entre los dos métodos, permitió evidenciar una subestimación del 9,6 \% de casos con lesión a través radiografia periapical respecto a la tomografia cone beam. De forma similar, Estrela y cols reportaron en un total de 1020 dientes, una frecuencia del 38,9\% de periodontitis apical a través de radiografía periapical y de 60,2\% con tomografía (7). Low y colaboradores realizaron un estudio con 74 dientes posteriores superiores e inferiores totalizando 156 raíces, a través de la tomografia detectaron 34 \% más de lesiones periapicales en comparación con las radiografías intrabucales (8). La concordancia obtenida entre los dos métodos evidencia una estimación de grado moderado, que deriva en el porcentaje de subestimación de lesiones observado en la radiografia periapical. 
El mayor porcentaje de pacientes de sexo masculino, observado en el presente estudio, concuerda con lo reportado en diversas investigaciones como el de mayor afectación de enfermedades periapicales (9-11). En este sentido, Mazarro y Chocarro (12) afirman que las mujeres dan mayor importancia a la salud y estética dental en comparación a los hombres, lo cual deriva en una mayor frecuencia de visitas al profesional de la salud bucal y consecuentemente una mayor oportunidad de diagnostico y tratamiento de casos de enfermedad dental. La edad como factor asociado a la enfermedad pulpar y periaplical permite identificar un mayor porcentaje de estas condiciones a medida en que el individuo avanza en su edad biológica. Lo anterior se confirma con lo concluido por Zavala y colaboradores (13) en su estudio sobre la prevalencia de enfermedades pulpares y periapicales en adultos mayores de la ciudad de Merida-Mexico y lo evidenciado en el estudio de Rincón (14) sobre la caraterización de pacientes asistentes a endodoncia, donde el 68,6 \% de ellos fue mayor de 40 años. De forma similar, en el presente estudio se observó una edad promedio de 45 años para los pacientes incluidos.

La mayor frecuencia de lesiones apicales en dientes multirradiculares del presente estudio, diagnosticadas por tomografia, converge con lo reportado por por Kabak y Abbott (15) en el estudio de prevalencia de periodontitis periapical en una población de 1423 adultos de Bielorrusia, donde esta condición fue más frecuente en dientes molares y premolares (37\%) (16).

Los estudios de éxito y fracaso en el tratamiento de endodoncica muestran que se obtiene un éxito que varia entre autores, y según las condiciones clínicas, y operatorias. Se ha demostrado que el porcentaje de éxito de la terapia endodontica está influenciado por la presencia o ausencia de radiolucides apical del diente a tratar endodonticamente. Es así que Sjögren et al., en 1990, encontraron que el porcentaje de éxito en general de la terapia endodóntica fue del 91 \% en pulpas vitales el porcentaje de éxito fue del 96, en raíces con necrosis pulpar y con lesión apical el porcentaje de éxito fue del 86 y en los dientes que se hizo necesario realizarle retratamiento endodóntico el porcentaje de éxito fue del 62 \% cuando la endodoncia se encuentra a nivel del apice radiográfico (17). En otro estudio realizado por De Quadros et al., en el 2005, reportan que el éxito encontrado en dientes con pulpas vitales estuvo entre un 81,8 \% y 96,3 \% después de un año de realizada la terapia endodóntica. Sin embargo, tres años después, esos dientes presentaron solamente un 60 de éxito tal vez debido a que no recibieron tratamientos protésicos adecuados (18).

Como hallazgos radiográficos observados en la evaluación de tomografia cone beam (tabla 2) la compresión del canal mandibular fue la que mas se presentó con un $30 \%$ de los hallazgos encontrados (19). Resultados similares a los que obtuvo 
Eliana Pineda Vélez, Samantha Bedoya Morales, Frank David Echeverri Romero, 11 Yeison Andrés Guerra Bedoya, Luisa Osorno Jaramillo, John Querubín Franco Aguirre

Montevecchi en el 2004, el cual realizó una investigación de 73 dientes terceros molares, de los cuales 14 presentaron estrechamiento del canal mandibular por compresión (20). La reabsorción radicular y la erosión de la tabla ósea vestibular fueron otros de los hallazgos encontrados con un 20 \% debido a que las lesiones endoperio por su cercana función embriónica y anatómica una puede llevar a la otra (21). La obturación parcial ocupo un 10 \% bajo a comparación de un estudio realizado por Webb en el 2017 dondel el 52\% de los dientes evaluados estaban suboturados (22). El rebalse apical, la pérdida de corona y la obturación en falsa vía también ocuparon cada uno un 10 \% entre los hallazgos. Estos eventos son comunes y pueden suceder cuando se hacen terapias endodónticas (23).

Como principal limitación del presente estudio, se considera el bajo tamaño de la muestra lo cual pudo derivar en una baja potencia en algunas de las pruebas de análisis bivariado y, consecuentemente, una mayor probabilidad de error tipo II y de sesgo de estimación en la evaluación de concordancia de los dos métodos aplicados.

\section{Conclusión}

Es de gran importancia profundizar en la necesidad de complementar la evaluación de los pacientes endodonticos con la ayuda diagnóstica de la tomografia cone beam, ya que es una herramienra que aun no es muy utilizada ni reconocida por los clínicos en su potencial diagnóstico, de plan de tratamientos y pronósticos de casos endodonticos.

\section{Referencias}

1. Boveda ZC, López GJ, Clavel DT. Tomografía Volumétrica Digital. 2012. Recuperado en: https://www.carlosboveda.com/tvd.htm

2. Maini A, Durning P, Drage N. Resorption: within or without? The benefit of cone-beam computed tomography when diagnosing a case of an internal/external resorption defect. Br Dent J. 2008; 204(3): 135-7.

3. Burgos Ponce J, Guimarães BM, Pinto LC. Tomografia computadorizada cone beam en el diagnóstico endodóntico - relato de un caso clínico. Parag Oral Res. 2012; 1: 39-42. 
Caracterización de dientes en una población con tratamiento de endodoncia atendida en una clínica odontológica universitaria mediante tomografía cone beam y comparación con radiografía periapical

4. De Paula-Silva FWG, Wu M-K, Leonardo MR, da Silva LAB, Wesselink PR. Accuracy of periapical radiography and cone-beam computed tomography scans in diagnosing apical periodontitis using histopathological findings as a gold standard. J Endod. 2009; 35(7): 1009-12.

5. Miyagaki DC, Marion J, Randi Ferraz CC. Diagnosis of vertical root fracture with cone-beam computerized tomography in endodontically treated teeth: three case reports. Iran Endod J. 2013; 8(2): 75-9.

6. Afrashtehfar KI. Utilización de imagenología bidimensional y tridimensional con fines Odontológicos. Rev Asoc Dent Mex. 2012; 69(3): 114-9.

7. Estrela C, Bueno MR, Porto OCL, Rodrigues CD, Pécora JD. Influence of intracanal post on apical periodontitis identified by cone-beam computed tomography. Braz Dent J. 2009; 20(5): 370-5.

8. Low KMT, Dula K, Bürgin W, von Arx T. Comparison of periapical radiography and limited cone-beam tomography in posterior maxillary teeth referred for apical surgery. J Endod. 2008; 34(5): 557-62.

9. Soberaniz-Morales V, Alonzo-Echeverría L, Vega-Lizama E. Frecuencia de patología pulpar en la clínica hospital de petróleos mexicanos Coatzacoalcos, Veracruz. Rev Científica Odontológica. 8(1): 7-111.

10. Fernández-Collazo ME, Vila-Morales D, Rodríguez-Soto A, Mesa-González DL, Pérez-Clemente NG. Lesiones periapicales agudas en pacientes adultos. Rev Cuba Estomatol. 2012; 49(2): 107-16.

11. Fernández-González M del C, Valcárcel-Llerandi J, Betancourt-Núñez M. Enfermedades pulpares y periapicales en trabajadores del Instituto Cubano de Oftalmología Ramón Pando Ferrer. Rev Habanera Cienc Médicas. 2009; 8(4): 0-0.

12. Mazarro-Bodega G, Chocarro-González L. Análisis de las diferencias de género en el cuidado bucodental. Gac Dent Ind Prof. 2012; (241): 114-26.

13. Zavala CE del PSM, Medina-Peralta S, Dorantes HHP. Prevalencia de enfermedades pulpares y periapicales en pacientes geriátricos: Mérida, Yucatán, México. Rev Cuba Estomatol. 2015; 52(3): 34-9.

14. Rincón-Forero LM. Caracterización de los pacientes asistentes a la clínica del posgrado de endodoncia de la Facultad de Odontología, Universidad Nacional de Colombia, 2010-2015 [Internet]. [Bogotá]: Universidad Nacional de Colombia; 2019 [citado 9 de julio de 2020]. Recuperado en: https://repositorio.unal.edu.co/handle/unal/58960 
Eliana Pineda Vélez, Samantha Bedoya Morales, Frank David Echeverri Romero, 13 Yeison Andrés Guerra Bedoya, Luisa Osorno Jaramillo, John Querubín Franco Aguirre

15. Kabak Y, Abbott PV. Prevalence of apical periodontitis and the quality of endodontic treatment in an adult Belarusian population. Int Endod J. 2005; 38(4): 238-45.

16. Ochoa L, Moreno S, Piarpuz\&aacute D, Rodriacute P, Herrera A, Moreno S. Evaluación del éxito y/o fracaso de los tratamientos de endodoncia en dientes no vitales realizados en la Escuela de Odontología de la Universidad del Valle. Serie de casos. Estomatología. 2014; 22(2): 13-20.

17. Sjogren U, Hagglund B, Sundqvist G, Wing K. Factors affecting the long-term results of endodontic treatment. J Endod. 1990;1 6(10): 498-504.

18. De Quadros I, Gomes BPFA, Zaia AA, Ferraz CCR, Souza-Filho FJ. Evaluation of endodontic treatments performed by students in a Brazilian Dental School. J Dent Educ. 2005; 69(10): 1161-70.

19. Kojima K, Inamoto K, Nagamatsu K, Hara A, Nakata K, Morita I, et al. Success rate of endodontic treatment of teeth with vital and nonvital pulps. A meta-analysis. Oral Surg Oral Med Oral Pathol Oral Radiol Endod. 2004; 97(1): 95-9.

20. Monaco G, Montevecchi M, Bonetti GA, Gatto MRA, Checchi L. Reliability of panoramic radiography in evaluating the topographic relationship between the mandibular canal and impacted third molars. J Am Dent Assoc 1939. 2004; 135(3): 312-8.

21. Pesqueira Cinco P, Carro Hernández H. Lesiones endoperiodontales. Odontol Vital. 2017; (27): 35-44.

22. Webb Porto D, Barrientos Sanchez S, Méndez De La Espriella C, Rodriguez Ciodaro A. Frecuencia y características de hallazgos endodónticos en radiografías panorámicas digitales. Odontoestomatología. 2017; 19(29): 76-84.

23. Terrazas Ríos TA, González Pérez G, Liñán Fernández M, Ortiz Villagómez M. Endodontic procedure accidents: Case report. Rev Odontológica Mex. septiembre de 2011; 15(3): 183-8. 